\title{
ESTUDIO SOBRE DOBLE PRESENCIA EN LA FACULTAD DE CIENCIAS DE LA EDUCACIÓN DE LA UNIVERSIDAD DE GRANADA
}

\author{
Lenka Krejčí, Aleksandra Łakoma, Aleksandra Niedziałkowska y Marta Nowotarska.
} Universidad de Granada.

\author{
Supervisado por la profesor José Miguel García Ramírez. Departamento de Psicología Social. Universidad de Granada \\ Fecha de recepción: 30 de mayo de 2014. \\ Fecha de revisión: 5 de junio de 2014. \\ Fecha de aceptación: 5 de julio de 2014.
}

\section{Resumen}

En el presente estudio investigamos sobre Doble presencia, que es uno de lo más importantes factores de los riesgos psicosociales. Es una necesidad de responder a las exigencias del trabajo y las responsabilidades domésticas sincrónicamente y es el balance sobre la vida y el trabajo que tiene que hacer una persona (Centro de Referencia de Organización del Trabajo y Salud, 2010). El objetivo de esta investigación fue analizar el factor Doble presencia para comprobar si afecta a la gente en el puesto de su trabajo. El estudio se realizó sobre una muestra de los profesores de la Facultad de las Ciencias de la Educación en la Universidad de Granada. Se usó el cuestionario Istas21 (CoPsoQ). Se encontró que Doble presencia presenta un problema grave y afecta principalmente a las mujeres. Al final del informe se sugieren algunas soluciones como se podría resolver el problema del factor analizado.

Palabras clave: Factores de Riesgo Psicosocial; Doble presencia; Educación Superior.

El trabajo es la base y fundamento de la vida social e individual. Es la actividad mediante la cual el hombre se relaciona con la naturaleza para satisfacer sus necesidades y desarrollarlas (Castillo, Santana, Valeta, Alvis, Romero, 2011). El estrés laboral puede afectar negativamente en la salud física y psicológica de un individuo, así como en la eficacia de una organización. Por lo tanto, la investigación en el campo de esta problemática se reconoce a nivel mundial como un gran reto para la salud de los trabajadores y la salud de sus organizaciones.

Para muchas personas trabajadoras es demasiado frecuente que el lugar de trabajo es el sitio en el que pasan la mayor parte de sus horas despiertos. De acuerdo con varias encuestas, muchos realizan actividades que ellos perciben como exigentes, restrictivas, y por lo demás estresantes. Problemas de salud mental y otros trastornos relacionados con el estrés son reconocidos como unas de las principales causas de molestias y trastornos músculo-esqueléticos, cese anticipado del trabajo, las altas tasas de ausencia y la baja productividad de la organización. Por eso es muy importante reconocer la importancia de los riesgos psicosociales (European Risk Observatory Report, 2012).

La Organizacion Intenacional del Trabajo (1986) define los riesgos psicosociales como: " las interacciones entre el contenido, la organización y la gestión del trabajo y las condiciones ambientales, por un lado, y las funciones y necesidades de los trabajadores/as, por otro. Estas interacciones podrían ejercer una influencia nociva en la salud de los trabajadores/as a través de sus percepciones y experiencias" (Secretaria de Accion Sindical, Salud Laboral y Medio Ambiente, 2006). 
Según Moncada et al. (2005) los riesgos psicosociales son características de las condiciones de trabajo y, concretamente, de la organización del trabajo nocivas para la salud. Existen cuatro grandes grupos de riesgos psicosociales que se pueden identificar en un puesto de trabajo a través del cuestionario Istas21; el exceso de exigencias psicológicas del trabajo, la falta de control sobre los contenidos y las condiciones de trabajo y de posibilidades de desarrollo, la falta de apoyo social, de calidad de liderazgo, de previsibilidad o de claridad de rol en el trabajo y las escasas compensaciones del trabajo. Otro factor de riesgo podría presentar la doble presencia (combinación de tareas domésticas y trabajo que presenta una sobrecarga para el individuo).

A continuación se explicarán con más detalle cada uno de los factores previamente mencionados. Primer factor presentan las Exigencias psicológicas, las necesidades mentales y físicas del trabajo. Hablamos de este factor de riesgo, por ejemplo, cuando hay que trabajar muy rápido, intensamente, y cuando hay muchas exigencias emocionales en el trabajo. Apoyo social y calidad de liderazgo forma el apoyo que los trabajadores reciben de compañeros de trabajo y son el grado en que los trabajadores perciben a sus superiores inmediatos para ser buenos líderes. Inseguridad pueden evocar los contratos precarios en el contexto de un mercado laboral inestable y transmitir una sensación de la inseguridad laboral. Estima sería el feedback que reciben los trabajadores de sus colegas y superiores inmediatos.

Doble presencia es una necesidad de responder a las exigencias del trabajo y las responsabilidades domésticas sincrónicamente. Es el balance sobre la vida y el trabajo. (Centro de Referencia de Organización del Trabajo y Salud, 2010). Muchos trabajadores deben combinar sus responsabilidades laborales con las tareas domésticas.

Las mujeres trabajadoras en particular, son responsables y hacen la mayor parte del trabajo doméstico y el cuidado de su familia. Además de sus obligaciones domésticas tienen un empleo. Por esta razón pueden ser más vulnerables a los riesgos psicosociales en comparación con los hombres (Mansilla, 2011). Estas desigualdades entre hombres y mujeres en cuanto a las condiciones de trabajo y la cantidad de trabajo realizado se manifiestan en desigualdades de salud entre hombres y mujeres. Esta "doble jornada" es en realidad una "doble presencia", porque las demandas tanto de puestos de trabajo como la productividad y la familia y el hogar, se toman diariamente sincrónicamente, ya que ambos requisitos coexisten simultáneamente. Investigación de Morenoa, Moncada, Llorensa, Carrasquerb (2010) ha demostrado una asociación entre doble presencia y las exigencias de alargamiento de jornada, los turnos rotativos y el horario irregular y la exposición a algunos riesgos psicosociales (las exigencias cuantitativas y emocionales altas). La doble presencia se debe considerar como una variable en la evaluación de riesgos psicosociales y las organizaciones deberían tomar medidas que tengan un impacto positivo sobre el mismo

El objetivo de este estudio fue identificar doble presencia en el personal de la Facultad de las Ciencias de Educación de la Universidad de Granada.

\section{Método}




\section{Participantes}

En el estudio participaron 87 personas, 34 hombres y 53 mujeres. La mayoría se encuentra en el grupo de edad 46-55 años, en el segundo grupo se encuentran las personas de 25-35 años y en tercer grupo personas de edad entre 36-45 años. La mayoría son las personas de la Facultad de Ciencias de la Educación (son 66 personas que forman el $76 \%$ ). Al respecto a antigüedad los datos varían mucho, hay una diferencia 37 años entre los más y menos antigüedad presentan.

\section{Instrumento}

Istas21 (CoPsoQ). El cuestionario mide las 6 dimensiones psicosociales que se encuentran en el puesto de trabajo. Estos son: Exigencias psicológicas, Trabajo activo y posibilidades de desarrollo, Inseguridad, Apoyo social y calidad de liderazgo, Doble presencia y Estima. Dependiendo de la puntuación obtenida cada una de estas dimensiones puede situarse en uno de los tres intervalos: verde, amarillo o rojo. A saber, para el intervalo verde la puntuación se sitúa entre 0 y 7 ; para el intervalo amarillo es de 8 a 10; para el intervalo rojo se encuentra entre 11 y 24 . Asimismo se inculyen las significaciones de cada de los tres: El intervalo verde es el nivel de exposición psicosocial más favorable para la salud; El intervalo amarillo es el nivel de exposición psicosocial intermedio; El inrevalo rojo es el nivel de exposición psicosocial más desfavorable para la salud.

\section{Procedimiento}

Administramos el cuestionario Istas21 a (como mínimo) dos personas cada uno de los alumnos con el fin de investigar su situación laboral actual y posibles riesgos psicosociales que podría padecer. A continuación se formaron grupos de alumnos que se especializaron en un riesgo concreto y elaboraron un informe en formato de un artículo científico de los datos encontrados sobre el mismo.

\section{Resultados}

Para comenzar a analizar los resultados obtenidos al principio se mencionarán las preguntas relacionadas con la variable Doble presencia. Las preguntas son siguientes:

- Pregunta 31 - hace referencia a que parte del trabajo y domestico hace la persona

- Pregunta 32 - hace referencia a que si la persona falta algún día de casa, si las tareas domésticas que realiza se quedan sin hacer

- Pregunta 33 -cuando está en la empresa: ¿piensa en las tareas domésticas y familiares?

- Pregunta 34 - si hay momentos en los que necesitaría estar en la empresa y en casa a la vez

Los resultados de la presente investigación de la muestra examinada se encuentran en el intervalo rojo que resulta de la media

\begin{tabular}{|r|c|c|c|c|c|}
\cline { 2 - 6 } \multicolumn{1}{c|}{} & Pregunta 31 & Pregunta 32 & Pregunta 33 & Pregunta 34 & Suma \\
\hline media & 3 & 2,022988506 & 1,471264368 & 1,597701149 & 8,091954023 \\
\hline desviación estándar & 0,940064322 & 1,275685055 & 1,043686227 & 1,05057869 & 3,262301171 \\
\hline
\end{tabular}

En este punto de análisis cabe mostrar la diferencia de la frecuencia de los tres intervalos: rojo, amarillo y verde. 
- El intervalo verde es el nivel de exposición psicosocial más favorable para la salud.

- El intervalo amarillo es el nivel de exposición psicosocial intermedio.

- El inrevalo rojo es el nivel de exposición psicosocial más desfavorable para la salud.

Como se puede observar en la siguiente tabla, la mayoría de las personas se sitúa en el intervalo rojo, es decir 59 de 87 personas que es casi $68 \%$. A su vez, la menor cantidad de personas reciben el intervalo verde, solamente 8 personas que son $9,2 \%$.

\begin{tabular}{|l|l|l|l|l|}
\hline Grado de importancia & Frecuencia & Porcentaje & $\begin{array}{l}\text { Por ciento de la } \\
\text { importante }\end{array}$ & Por ciento de la acumulativa \\
\hline Verde & 8 & 9,2 & 9,2 & 9,2 \\
\hline amarillo & 20 & 23,0 & 23,0 & 32,2 \\
\hline Rojo & 59 & 67,8 & 67,8 & 100,0 \\
\hline Totales & 87 & 100,0 & 100,0 & \\
\hline
\end{tabular}

En el análisis siguiente se utilizó el chi-cuadrado que confirma que existe una relación importante entre genero y el nivel de Doble Presencia obtenida.

\begin{tabular}{|l|l|l|l|}
\hline & Valor & Df & Significancia asintótica \\
\hline Chi-cuadrado de Pearson & $9,077^{\star}$ & 2 &, 011 \\
\hline Credibilidad cociente & 9,046 & 2 &, 011 \\
\hline Prueba de agrupación lineal & 8,926 & 1 &, 003 \\
\hline Número de observaciones importantes & 87 & & \\
\hline
\end{tabular}

*El 33,3\% de las células (2) tiene un valo inferior a 5. Valor mínimo esperado es de 3.13

Los resultados difieren debido a género. Es decir, las mujeres reciben las puntuaciones más altas que los hombres para cada una de las preguntas.

\begin{tabular}{|l|l|l|l|l|l|l|}
\cline { 2 - 7 } \multicolumn{1}{c|}{} & & Pregunta 31 & Pregunta 32 & Pregunta 33 & Pregunta 34 & Suma \\
\hline \multirow{2}{*}{ MUJER } & media & 3,283018868 & 2,452830189 & 1,622641509 & 1,79245283 & 9,150943396 \\
\cline { 2 - 7 } & desviación estándar & 0,817532838 & 1,084258657 & 1,147345014 & 1,044371187 & 3,128013786 \\
\hline \multirow{2}{*}{ HOMBRE } & media & 2,558823529 & 1,352941176 & 1,235294118 & 1,294117647 & 6,441176471 \\
\cline { 2 - 7 } & desviación estándar & 0,959519339 & 1,276414318 & 0,818676816 & 1,000890869 & 2,776428668 \\
\hline
\end{tabular}

También recogimos resultados cualitativos: Los participantes de estudio, que se sitúan dentro del intervalo Rojo proponen algunas soluciones para resolver su problema con Doble Presencia. El análisis de su expresión, podemos ver que algunos adoptan una actitud pasiva ("nadie no puede ayudarme" "tengo que esperar a que mis hijas crezcan") y otros activa ("proponer rotación de las tareas" "delegar tareas a su marido" "mejorar organización de mi tiempo").

También vemos una tendencia, que algunas personas quieren mejorar la situación únicamente por su cuenta cuando otros buscan apoyo por parte de otros miembros de la familia. Los primeros proponen por ejemplo:

- "evitar tener que llevar el trabajo a casa"

- "planificar mejor el día"

- "mejorar organización de su tiempo"

- "dividir su atención según la tarea que está realizando"

- "realizar las tareas del hogar según la importancia y prioridad que tenga no dándole tanta importancia a las tareas menores"

Estas personas no piden ayuda por parte de otras personas. No están acostumbradas de compartir las tareas del hogar y no quieren (o no pueden) cambiarlo. 
La segunda parte de los encuestados admite que apoyo de otras personas pede mejorar su situación:

- "distribución de tareas domésticas con el resto de los miembros de la unidad familiar"

- "rotación de tareas en casa"

- "empleados domésticos para la ayuda en el hogar con las tareas"

- "de dividir las responsabilidades con su marido"

- "buscar a alguna persona de confianza que pudiera ayudarla con las tareas del hogar"

Las personas que pertenecen a cada uno de estos grupos perciben su problema en la manera diferente, por lo tanto, requieren una intervención diferente por parte de la psicóloga.

\section{Discusión}

Los resultados obtenidos en el presente estudio indican que el factor de la Doble Presencia es un problema para los empleados de la Facultad de Ciencias de la Educación en la Universidad de Granada. Parece que las mujeres en este sentido están significativamente más afectadas que los hombres. Es posible que la edad también influye en cierta medida, aunque no se encontró una diferencia significativa entre los distintos grupos de edad.

En este trabajo vamos a sugerir algunas posibles soluciones. Dado que en el problema de la doble presencia se ven implicados varios factores, consideramos que las medidas de mejora deberían venir tanto de la parte de la organización, como del propio hogar de la persona afectada. En el ámbito laboral sería importante mantener los tiempos de la jornada laboral sin prolongarlos de una forma abusiva y ajustar el nivel de las exigencias a un nivel posible de conseguir para la persona sin sobrecargarla. En el ámbito de la vida personal de la persona sería deseable que las tareas domésticas se repartieran de una forma equilibrada entre todos los miembros de la familia y que la persona aprenda a organizar de una forma eficaz su tiempo de trabajo y de descanso.

En cuanto a la metodología hay algunos aspectos en el presente estudio que no se han controlado y que han podido influir en los resultados. En esta investigación participaron más mujeres que hombres. Cómo indican los resultados hay una interacción entre el sexo y la doble presencia, por lo tanto podemos esperar que el número más elevado de las mujeres que el de los hombres ha podido influir. La edad de los participantes no está distribuida de una forma uniforme, en el tercer grupo de edad hubo muy pocos participantes. La antigüedad en el puesto tampoco está distribuida de manera uniforme. La mayoría, en concreto el setenta y seis por cien de los participantes, son las personas de la Facultad de Ciencias de la Educación. En la interpretación de los resultados numéricos individuales no se tienen en cuenta las excepciones que pueden tener lugar en algunos casos. En los próximos estudios sería conveniente controlar las variables de una forma más rigurosa, contar con las excepciones en las interpretaciones de las puntuaciones y administrar el cuestionario en más facultades.

A modo de conclusión, esta corriente de investigación permite acercarse al profesorado a través de la simpatía (Garcia-Ramirez, 2012), de esa manera se puede indagar sobre sus condiciones de trabajo y cómo estas influyen en la calidad de su vida laboral; también ayuda a identificar posibles riesgos psicosociales que afectan al 
profesorado y a la búsqueda de soluciones para conseguir el bienestar tanto en el profesorado como en la organización.

\section{Referencias}

Centro de Referencia de Organización del Trabajo y Salud (2010). Instituto Sindical de Ambiente, Trabajo y Salud. Manual del método CoPsoQ-istas21 (versión 1.5) para la evaluación y prevención de los riesgos psicosociales para empresas con 25 o más trabajadores y trabajadoras. Barcelona: Instituto Sindical de Trabajo, Ambiente y Salud.

Castillo IY, Santana M, Valeta A, Alvis LR, Romero E.( 2011). Factores de riesgo psicosociales del trabajo en médicos de una Empresa Social del Estado en Cartagena de Indias, Colombia. Rev. Fac. Nac. Salud Pública, 29(4): 363-371

European Commission (2008). Employment In Europe report, Office for Official Publications of the European Communities, Luxembourg.

European Risk Observatory Report (2012). Management of psychosocial risks at work: An analysis of the findings of the European Survey of Enterprises on New and Emerging Risks. European Agency for Safety and Health at work.

García-Ramírez, J.M. (2012). La comunicación, clave de excelencia visible en la Educación Superior. Journal for Educators, Teachers and Trainers, Vol. 3, pp. 25-36. http://www.ugr.es/ jett/pdf/vol03_02_jett_garcia-ramirez.pdf.

ISTAS. Instituto sindical de trabajo ambiente y salud. Doble presencia. http://www.istas.net/web/index.asp?idpagina=1979.

Mansilla F (2011). La evaluación de riesgos psicosociales en el trabajo y la planificación de la actividad preventiva. Psicología online. http://www.psicologia-online.com/ebooks/riesgos/capitulo2_2.shtml.

Moncada, S., Llorens, C., Navarro, A. y Kristensen, T.S. (2005). ISTAS21: Versión en lengua castellana del cuestionario psicosocial de Copenhague (COPSOQ). Archivos de Prevención de Riesgos Laborales, 8(1) 18-29.

Morenoa N., Moncada S., Llorensa C., Carrasquerb P., (2010). Doble presencia, trabajo doméstico- familiar y asalariado: espacios sociales y tiempos. Instituto Sindical de Trabajo, Ambiente y Salud. Universitat Autònoma de Barcelona.

Secretaria de Accion Sindical, Salud Laboral y Medio Ambiente (2006). Definicion de los resgos psicosociales. Manual de riesgos psicosociales en el mundo laboral, UGT Aragon.

Wellnomics (2008). Psychosocial risk factors: what are they and why are they important. http://www.workpace.com/assets/Uploads/White-Papers/WellnomicsWhite-paper-Psychosocial-risk-factors-What-are-they-and-why-are-theyimportant.pdf. 